\title{
Generation of porcine fetal fibroblasts expressing the tetracycline-inducible Cas9 gene by somatic cell nuclear transfer
}

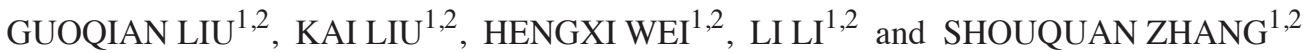 \\ ${ }^{1}$ Guangdong Provincial Key Lab of Agro-Animal Genomics and Molecular Breeding; \\ ${ }^{2}$ National Engineering Research Center For Breeding Swine Industry, College of Animal Science, \\ South China Agricultural University, Guangzhou, Guangdong 510642, P.R. China
}

Received March 26, 2015; Accepted February 25, 2016

DOI: $10.3892 / \mathrm{mmr} .2016 .5530$

\begin{abstract}
Cas9 endonuclease, from so-called clustered regularly interspaced short palindromic repeats (CRISPR)/CRISPR-associated (Cas) systems of Streptococcus pyogenes, type II functions as an RNA-guided endonuclease and edits the genomes of prokaryotic and eukaryotic organisms, including deletion and insertion by DNA double-stranded break repair mechanisms. In previous studies, it was observed that Cas9, with a genome-scale lentiviral single-guide RNA library, could be applied to a loss-of-function genetic screen, although the loss-of-function genes have yet to be verified in vitro and this approach has not been used in porcine cells. Based on these observations, lentiviral Cas9 was used to infect porcine primary fibroblasts to achieve cell colonies carrying Cas9 endonuclease. Subsequently, porcine fetal fibroblasts expressing the tetracycline-inducible Cas9 gene were generated by somatic cell nuclear transfer, and three 30 day transgenic porcine fetal fibroblasts (PFFs) were obtained. Polymerase chain reaction (PCR), reverse transcription-PCR and western blot analysis indicated that the PFFs were Cas9-positive. In addition, one of the three integrations was located near to known functional genes in the PFF1 cell line, whereas neither of the integrations was located in the PFF1 or PFF2 cell lines. It was hypothesized that these transgenic PFFs may be useful for conditional genomic editing in pigs, and for generating ideal modified porcine models.
\end{abstract}

\section{Introduction}

Pigs are one of the most important large animal models for xenotransplantation associated with various human diseases,

Correspondence to: Professor Shouquan Zhang, Guangdong Provincial Key Lab of Agro-Animal Genomics and Molecular Breeding, College of Animal Science, South China Agricultural University, 483 Wushan Road, Guangzhou, Guangdong 510642, P.R. China

E-mail: sqzhang@scau.edu.cn

Key words: pig, fibroblasts, somatic cell nuclear transfer, cas9 endonuclease, cell lines since pigs are physiologically similar to humans and are widely available (1). Thus, pigs serve as a biomedical animal model, and they have been genetically modified (GM) and primarily produced using the technique of somatic cell nuclear transfer (SCNT) (2). Until recently, most genetically altered pigs were generated by random insertions of expression cassettes into porcine fetal fibroblasts (PFFs), or by editing the genome (3). Zinc finger nucleases (4-6) and transcription activator-like effector nucleases, or 'TALENs' (7-9), have provided powerful tools to generate specific gene modifications in pigs; however, the complex design and generation greatly limit the application of these systems. The so-called clustered regularly interspaced short palindromic repeats (CRISPR) system, relying on CRISPR RNAs (crRNAs) in combination with CRISPR-associated (Cas) proteins to direct the degradation of complementary sequences present within invading viral and plasmid DNA, has been demonstrated to be an alternative strategy for precise gene editing $(10,11)$. Cas9 endonuclease, from the Streptococcus pyogenes type II CRISPR/Cas system, functions as an RNA-guided endonuclease that uses a dual-guide RNA, consisting of crRNA and trans-activating crRNA (tracrRNA), for target recognition and cleavage by a mechanism involving two nuclease active sites that together generate double-stranded DNA (dsDNA) breaks, which requires the recognition of a short trinucleotide proto-spacer adjacent motif (PAM) sequence (-NGG) following the 20 bp crRNA target (12).

RNA-guided Cas9 nucleases have been successfully used to generate GM pigs via the direct cytoplasmic injection of Cas9 mRNA and single guide (sg)RNA into zygotes $(13,14)$, or through the modification of somatic cells followed by SCNT (15). The CRISPR/Cas9 system has been applied to genome-wide loss-of-function screening in human cells $(16,17)$ and mouse embryonic stem cells $(18)$, and it is superior as a method to RNA interference (RNAi); however, the loss-of-function genes have not been verified in vitro and the approach has not been used in pig cells, particularly in primary cells. Additionally, for certain genes that are lethal to embryos, it is difficult to generate knockouts to study other functions.

In the present study, lentiviral cas 9 was used to infect pig primary fibroblasts in order to generate cell colonies carrying the Cas9 protein. Subsequently, SCNT from pools of stably nucleofected cell clones was used to generate inducible 
transgenic expression cell lines for Cas9 protein. Thus, the Cas9 cell lines are a convenient and useful tool for editing the pig genome and for establishing pig models.

\section{Materials and methods}

Animal and recombinant DNA usage. The use of animals was approved by the Animal Care and Use Committee of the South China Agricultural University, Guangzhou, China. Recombinant DNA use was approved by the Institutional Biosafety Committee. All surgical procedures were performed under anesthesia, and all efforts were made to minimize animal suffering.

Guide (g)RNA design and plasmid construction. The lentiviral doxycycline-inducible FLAG-Cas9 (50661) and U6-sgRNA cloning vector (50662) plasmids were purchased from Addgene, Inc. (Cambridge, MA, USA). Targeting sgRNA was designed as previously described (16). An extra guanine was added to the 5 ' end of the gRNA in which the first nucleotide was not guanine for more efficient transcription by RNA polymerase III (19). These constructs were confirmed by sequence analysis.

Virus production, transduction and selection. Lentivirus of FLAG-Cas9 was produced by co-transfection of the lentiviral transfer vector with the pMD2.G and pPAX2 packaging plasmids (Addgene, Inc.) into 293FT cells using Lipofectamine 3000 transfection reagent (Invitrogen Life Technologies; Thermo Fisher Scientific, Waltham, MA, USA). The virus-containing supernatant was collected 48 and $72 \mathrm{~h}$ following transfection, cleared of cell debris by filtering through a $0.45 \mu \mathrm{m}$ filter and concentrated by ultracentrifugation (Merck Millipore, Darmstadt, Germany) at 4,000 g for $30 \mathrm{~min}$ at $4^{\circ} \mathrm{C}$. PFFs were cultured until they had reached 50-80\% confluence in six-well tissue culture plates, and were subsequently infected in media containing $10 \mu \mathrm{g} / \mathrm{ml}$ polybrene (Sigma-Aldrich, St. Louis, MO, USA) and concentrated lentivirus. At $12 \mathrm{~h}$ following infection, the virus was removed and cells were selected with $2 \mu \mathrm{g} / \mathrm{ml}$ puromycin (Sigma-Aldrich) for 5-7 days. Cell colonies were picked and cultured in 48-well plates. After 2 days, the cell colonies were subcultured and selected with $1 \mu \mathrm{g} / \mathrm{ml}$ puromycin for an additional 10 days. Subsequently, a fraction of selected cells was used for polymerase chain reaction (PCR) and reverse transcription (RT)-PCR detection, and the other cells were frozen for future use.

$S C N T$. Porcine SCNT was performed as described previously $(15,20)$. Briefly, cumulus-oocyte complexes were aspirated from the ovaries and matured in maturation medium for $42-44 \mathrm{~h}$ at $39^{\circ} \mathrm{C}$, as described previously (21). Cumulus cells were removed by repeated pipetting in $0.1 \%$ hyaluronidase (Sigma-Aldrich). Matured oocytes with a first polar body were enucleated manually in the presence of $7.5 \mathrm{mg} / \mathrm{ml}$ cytochalasin B (Sigma-Aldrich). A single fibroblast cell that was identified for gene integration by genotyping was microinjected into the perivitelline space of the oocytes. The oocyte-donor cell complexes were cultured in PZM3 medium (21) at $39^{\circ} \mathrm{C}$ for $1.5 \mathrm{~h}$, and subsequently fusion and activation were performed with two successive direct current pulses at $1.2 \mathrm{kV} / \mathrm{cm}$ for $30 \mu \mathrm{s}$ using an electrofusion instrument (model, CF-150/B; BLS Ltd., Budapest, Hungary). The reconstructed embryos were cultured in PZM3 medium at $39^{\circ} \mathrm{C}$ for $20 \mathrm{~h}$ and surgically transferred to the oviducts of the embryo recipients that were estrous-synchronized. The pregnancy status of the recipient sows was monitored by ultrasonography at $\sim 25$ days following the embryo transfer.

Cas9-PFF generation and culture. PFFs were isolated from 30-day-old fetuses of cloned pigs that were integrated with the Cas9 gene by tissue explant culture, as described previously (22). First, the fetuses were washed in phosphate buffered saline (PBS) containing $100 \mathrm{U} / \mathrm{ml}$ penicillin and $100 \mathrm{mg} / \mathrm{ml}$ streptomycin (Sigma-Aldrich China, Inc., Shanghai, China), and subsequently the heads, tails, limbs and viscera were removed under sterile conditions. Secondly, the residual tissues were washed twice in PBS and cut into small pieces $\left(\leq 1 \mathrm{~mm}^{3}\right)$, pasted on to $100-\mathrm{mm}$ cell culture dishes, and $5 \mathrm{ml}$ fetal bovine serum (FBS) was subsequently added. The culture dishes were incubated in humidified $95 \%$ air with $5 \% \mathrm{CO}_{2}$ at $39^{\circ} \mathrm{C}$ for $24 \mathrm{~h}$. Typically, the first outgrowing cells from the fetal explants became visible within $24 \mathrm{~h}$ of incubation, at which point the medium was replaced with Dulbecco modified Eagle's medium (DMEM) supplemented with $2 \mathrm{mM}$ glutamine, 100 units $/ \mathrm{ml}$ penicillin and $100 \mathrm{mg} / \mathrm{ml}$ streptomycin containing 10\% FBS (Gibco $^{\mathrm{TM}}$; Thermo Fisher Scientific) for 2-3 days. The cultured cells were expanded and subsequently cryopreserved for future use.

$P C R$ and RT-PCR detection. Genomic DNA was isolated from selected cell colonies or Cas9-PFFs and wild-type cells using a TIANamp Genomic DNA kit [Tiangen Biotech (Beijing) Co., Ltd, Beijing, China]. The total RNA of the cell colonies or Cas9-PFFs induced with $1 \mu \mathrm{g} / \mathrm{ml}$ doxycycline for $48 \mathrm{~h}$ was extracted using an E.Z.N.A. ${ }^{\circledR}$ Total RNA kit (Omega Bio-Tek, Inc., Norcross, GA, USA), following the manufacturer's protocol and including DNase I (Takara Biotechnology Co., Ltd., Dalian, China) treatment to remove the genomic DNA. Complementary DNA (cDNA) was synthesized at $42^{\circ} \mathrm{C}$ for 60 min using a PrimeScript ${ }^{\mathrm{TM}}$ RT-PCR kit (Takara Biotechnology Co., Ltd.) with oligo (dT) primers. PCR was performed for genomic lever and RT-PCR for gene transcripts of Cas9 in a programmed thermal cycler (Bio-Rad Laboratories, Inc., Hercules, CA, USA). The following PCR and RT-PCR conditions were used: Initial denaturation at $94^{\circ} \mathrm{C}$ for $3 \mathrm{~min}$, followed by 35 cycles of denaturation at $94^{\circ} \mathrm{C}$ for $30 \mathrm{sec}$; annealing at $60^{\circ} \mathrm{C}$ for $30 \mathrm{sec}$ and extension at $72^{\circ} \mathrm{C}$ for $1 \mathrm{~min}$; and $10 \mathrm{~min}$ final extension at $72^{\circ} \mathrm{C}$.

Indirect immunofluorescent assay (IFA). Treated and untreated control cells cultured on cover slips were fixed with $4 \%$ paraformaldehyde for $10 \mathrm{~min}$, permeabilized with 0.5\% Triton X-100 (Sigma-Aldrich) for $15 \mathrm{~min}$, and blocked with $1 \%$ bovine serum albumin for $30 \mathrm{~min}$. After rinsing twice with PBS, a 1:200 dilution of the anti-FLAG monoclonal antibody (cat. no. AE005; ABclonal Biotechnology Co., Ltd., Cambridge, MA, USA) was added to the cells, incubated overnight at $4{ }^{\circ} \mathrm{C}$, and subsequently washed thrice in the washing buffer. The cells were then sequentially incubated with fluorescein isothiocyanate-conjugated goat anti-mouse secondary antibody (cat. no. 7076; Cell Signaling Technology 
Table I. Primer sequences.

\begin{tabular}{llll}
\hline Name & Direction & Description & Sequences $\left(5^{\prime}-3^{\prime}\right)$ \\
\hline Cas9-1f & Forward & Cas9 gene PCR & ACAAGCTGATCCGGGAAGTG \\
Cas9-1r & Reverse & and RT-PCR primers & CTGTCTGCACCTCGGTCTTT \\
$\beta$-actin-f & Forward & Pig actin detection & GTGCGGGACATCAAGGAGAA \\
$\beta$-actin-r & Reverse & RT-PCR primers & GTCACCTTCACCGTTCCAGT \\
LTR1 & Forward & First IPCR & GAGGGATCTCTAGTTACCAGAGTCACA \\
P1 & Reverse & Primers & GAAGAATCGCAAAACCAGCAAGAAAAG \\
LTR2 & Forward & Secondary IPCR & AGCCAGAGAGCTCCCAGGCTCAGATC \\
P2 & Reverse & Primers & CATAATGATAGTAGGAGGCTTGGTAGG
\end{tabular}

RT-PCR, reverse transcription-polymerase chain reaction; IPCR, inverse PCR; LTR, large untranscribed region.
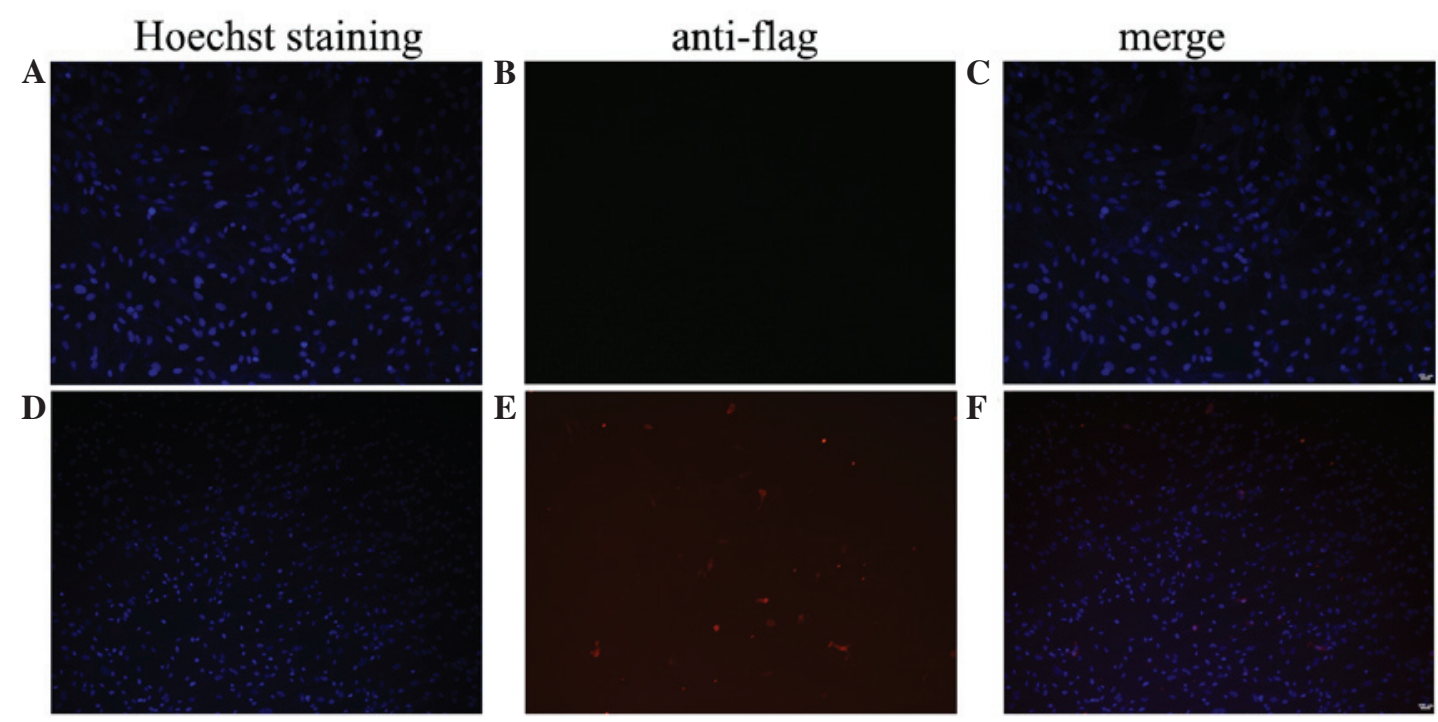

Figure 1. Doxycycline-inducible FLAG-Cas9 expression in porcine primary cells. (A-C) PFF-transfected Cas9 without doxycycline treatment for $48 \mathrm{~h}$. (D-F) PFF-transfected Cas9 with $1 \mu \mathrm{g} / \mathrm{ml}$ doxycycline treatment for $48 \mathrm{~h}$. PFF, porcine fetal fibroblast.

, Inc., Danvers, MA, USA) at a dilution of 1:50 for $1 \mathrm{~h}$ at $37^{\circ} \mathrm{C}$. Following washing, the cells were stained for DNA with $10 \mu \mathrm{g} / \mathrm{ml}$ Hoechst 33342 (Molecular Probes) and viewed under a fluorescent microscope (IX71; Olympus Corporation, Tokyo, Japan).

Western blotting. Protein extracts were obtained from Cas9 PFFs induced with $1 \mu \mathrm{g} / \mathrm{ml}$ doxycycline for $72 \mathrm{~h}$ and wild-type PFFs. The protein concentrations were determined using the Bradford method (23). Primary anti-FLAG monoclonal antibody (1:2,000 dilution; ABclonal Biotechnology) and anti-mouse immunoglobulin $\mathrm{G}$ horseradish peroxidase (1:3,000 dilution; Cell Signaling Technology, Danvers, MA, USA) were used for the western blot analysis. Western blotting was performed as previously reported (24).

Inverse PCR and integration analysis. To analyze FLAG-Cas9 insertion sites, $2 \mu \mathrm{g}$ transgenic pig genomic DNA was digested with XhoI, purified on a DNA purification column (Omega Bio-Tek, Inc.), and eluted with $20 \mu \mathrm{l}$ doubly distilled $(\mathrm{dd}) \mathrm{H}_{2} \mathrm{O}$. ElutedDNA was self-ligated in a $400 \mu \mathrm{l}$ reaction system, including
400 units $\mathrm{T} 4$ ligase at $16^{\circ} \mathrm{C}$ overnight, prior to being purified on a DNA purification column and eluted with $20 \mu \mathrm{lddH_{2 }} \mathrm{O}$. The resulting circular DNA underwent nested PCR, and was amplified using the specific primers, LTR-1+P1 and LTR-2+P2 (for primer sequences, see Table I). The PCR products were cloned into pMD-18T, a simple cloning vector (Takara Biotechnology Co., Ltd.) and sequenced. Sequences were aligned to the sequence of the donor vector, pCW-Cas9, and the Sus scrofa (wild pig) genomic DNA sequence database (Build Sscrofa10.2) using the National Center for Biotechnological Information's Basic Local Alignment Search Tool ('BLAST').

\section{Results}

Assessment of doxycycline-inducible FLAG-Cas9 in porcine primary cells. To determine doxycycline-inducible FLAG-Cas9 gene expression in porcine primary cells, the plasmid-expressed Cas9 was transfected into porcine fibroblasts and 293T cells as a control. Cas9 expression was confirmed at $48 \mathrm{~h}$ using an IFA. The results demonstrated that the tet-on system, which expresses FLAG-Cas9, is able to function properly in porcine 
Table II. Somatic cell nuclear transfer results for the generation of FLAG-Cas9 PFFS.

\begin{tabular}{lcccccc}
\hline Target gene & Cell pool & $\begin{array}{c}\text { Transferred } \\
\text { embryos }\end{array}$ & $\begin{array}{c}\text { No. } \\
\text { recipients }\end{array}$ & $\begin{array}{c}\text { No. (\%) } \\
\text { pregnancies }\end{array}$ & $\begin{array}{c}\text { No. 30 day } \\
\text { fetuses }\end{array}$ & $\begin{array}{c}\text { No. (\%) } \\
\text { transgenic } \\
\text { PFFs }\end{array}$ \\
\hline Cas9 & Clo1-4 & 280 & 1 & 0 & 0 & 0 \\
& Clo5-7 & 270 & 1 & 0 & 0 & 0 \\
& Clo8-11 & 285 & 1 & 1 & 3 & 3 \\
\hline
\end{tabular}

PFF, porcine fetal fibroblast.

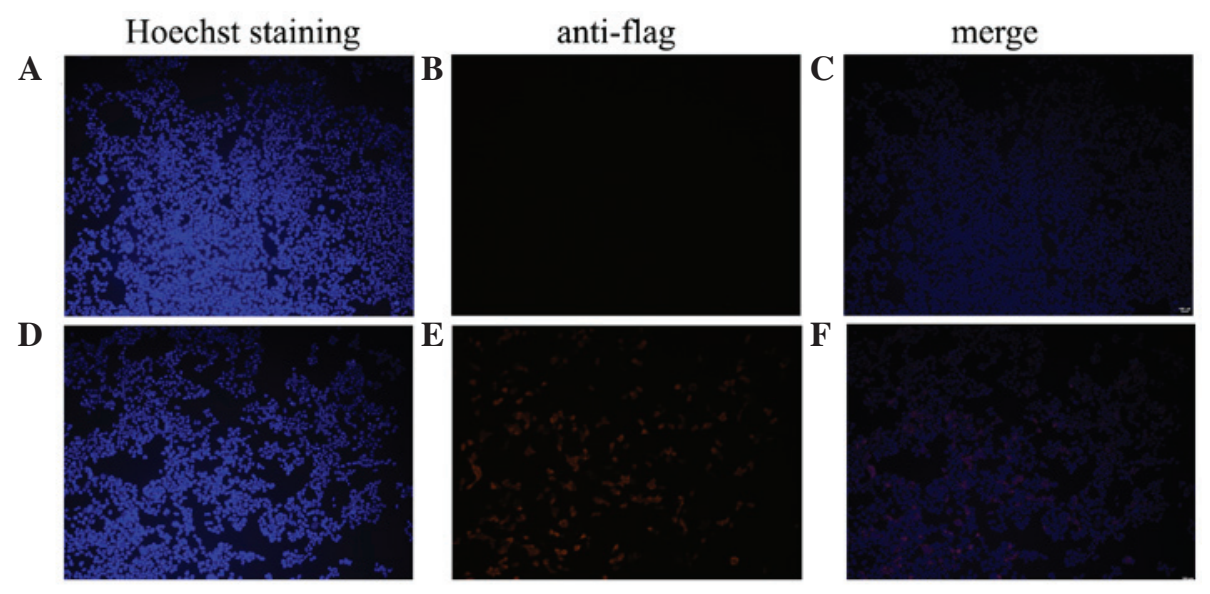

Figure 2. Doxycycline-inducible FLAG-Cas9 expression in 293T cells.(A-C) PFF-transfected Cas9 without doxycycline treatment for $48 \mathrm{~h}$. (D-F) PFF-transfected Cas9 with $1 \mu \mathrm{g} / \mathrm{ml}$ doxycycline treatment for $48 \mathrm{~h}$. PFF, porcine fetal fibroblast.

fibroblasts (Fig. 1), although it was weaker compared with $293 \mathrm{~T}$ cells (Fig. 2).

Generation of transgenic PFF colonies. Following selection and culture for 20 days, 16 PFF colonies were obtained (Fig. 3A), and whether or not the target gene had been integrated into the porcine genome was subsequently determined. The results of the genomic DNA PCR analysis revealed that all colonies had integrated the Cas9 gene (Fig. 3B).

Generation of FLAG-Cas9 PFFs via SCNT. Eleven transgenic PFF colonies containing the Cas9 gene were selected as donors for SCNT. A total of 835 reconstructed embryos were introduced into three surrogate mothers. Among the surrogate mothers, one was revealed to be pregnant by ultrasonography 25 days following embryo transfer, and the other two were not apparently pregnant (Table II). Therefore, the pregnant surrogate mother was sacrificed at 30 days, and three normal fetuses were obtained (Fig. 4A). The genomic DNA PCR analysis demonstrated three Cas9-positive PFFs (Fig. 4B). RT-PCR and western blot analyses indicated that three Cas9-positive PFFs successfully exhibited mRNA and protein expression of the Cas9 gene (Fig. 4C and D). However, the FLAG-Cas9 gene was expressed at a lower level in PFF1 compared with PFF2 and PFF3 (Fig. 4C).

Identification of FLAG-Cas9 integration sites in the transgenic PFFs genome. To analyze FLAG-Cas9 integration, inverse PCR was used to identify the lentiviral vector insertion sites in the genome of transgenic PFFs expressing Cas9. Three integration sites were identified in the transgenic PFF1 genome, integrated on chromosomes 2, 7 and 9 (Fig. 5A), one of which was located near to known functional genes. Two integration sites (on chromosomes 1 and 16) were identified in the transgenic PFF2 (Fig. 5B), and one integration site was identified in PFF3 (Fig. 5C), none of which were located near to known functional genes.

\section{Discussion}

Cas9 is a dsDNA endonuclease that uses a crRNA guide to specify the site of cleavage, and is an essential component of the CRISPR/Cas9 system $(11,25)$. The cell lines expressing Cas9 have provided a convenient system with which to generate targeted mutations of any gene by simple transfection with sgRNA (26). In the present study, three doxycycline-inducible flag-cas9 PFFs were successfully generated through SCNT, which conditionally expressed Cas9 endonuclease. Consistently with previous reports $(27,28)$, the transfection efficiency of the doxycycline-inducible FLAG-Cas9 vector was lower in porcine fibroblasts; however, transgenic PFF1 expressed less Cas9 protein compared with the other transgenic PFFs, which may be associated with integration sites on the PFF genome.

The CRISPR/Cas9 system was initially used to edit the genomes of prokaryotic and eukaryotic organisms, including deletion and insertion by DNA double-stranded break (DSB) 
A

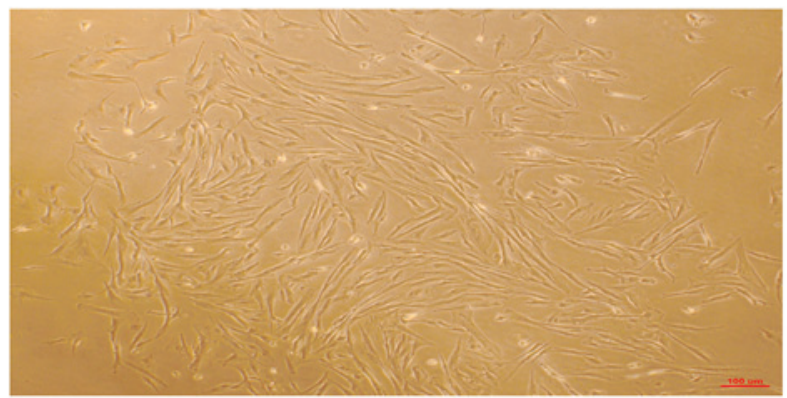

\section{B}

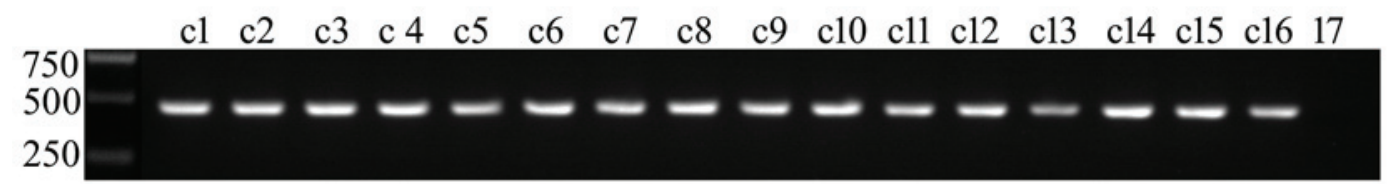

Figure 3. Generation of transgenic PFF colonies. (A) PFF clones after 7 days with $2 \mu \mathrm{g} / \mathrm{ml}$ of puromycin selection. Red bar=100 $\mu \mathrm{m}$. (B) Detection of the Cas9 gene at the genomic level by polymerase chain reaction. c1-c16 represent transgenic PFF colonies with integrated Cas9 gene; c17 is the wild-type PFF colony. PFF, porcine fetal fibroblast.

A

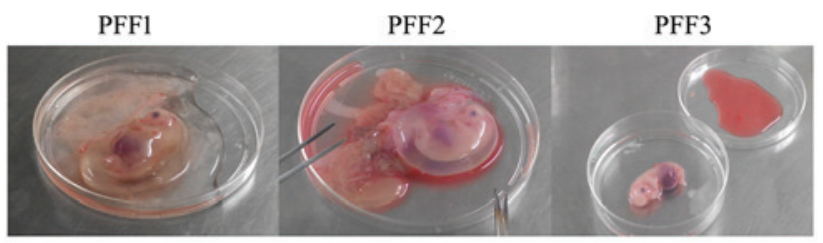

C

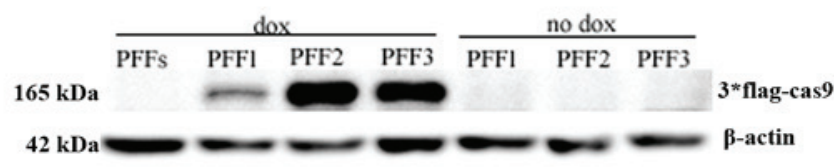

B

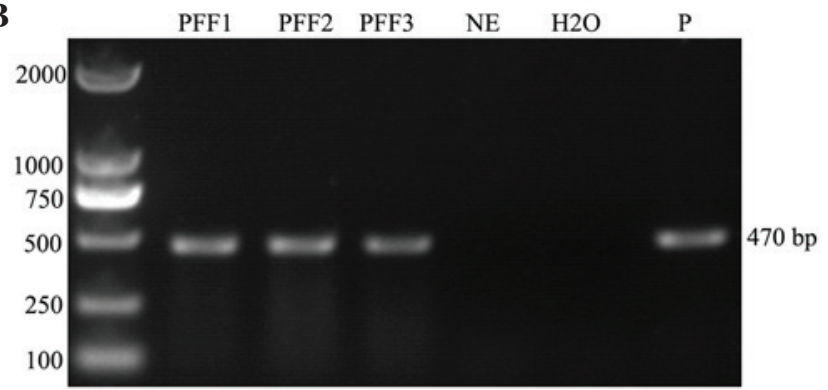

D

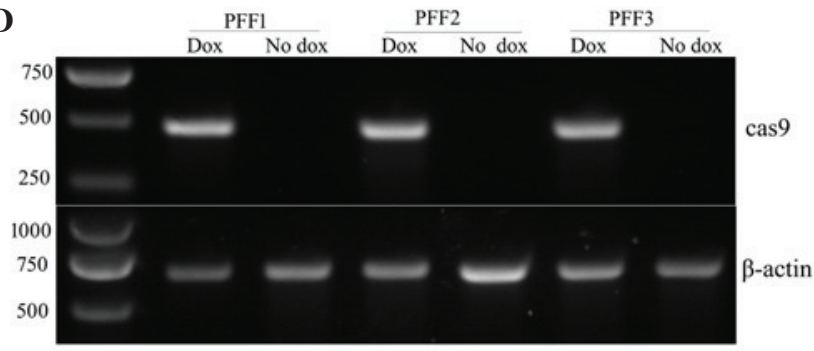

Figure 4. Generation of FLAG-Cas9PFF. (A) Generation of three clonal 30 day fetuses. (B) Detection of the Cas9 gene of the clonal fetuses by PCR. (C) Expression of Cas9-PFFs. Clonal Cas9-PFFs were induced by dox for $48 \mathrm{~h}$, and Cas9 protein was detected using FLAG-tag protein. (D) Detection of the Cas9 gene of the clonal fetuses at the transcriptome level by RT-PCR. Three clonal PFFs were treated with dox for $48 \mathrm{~h}$, and untreated as the control. PFF, porcine fetal fibroblast; NE, untreated porcine embryo; dox, doxycycline; RT-PCR, reverse transcription-polymerase chain reaction.

repair mechanisms. Cas9 endonuclease, with sgRNAs, was shown to be able to modify single $(29,30)$ or multiple (31) genes. Previously, the CRISPR/Cas9 system has been used to create pigs of one genetic strain or multiple genetic modifications in a single pregnancy (32); however, the present study aimed to directly generate modified organisms using Cas9 endonuclease and targeted sgRNAs. In the present study, PFFs were generated expressing stable tetracycline-regulated Cas9 endonuclease. Subsequently, the genes of interest were specifically knocked out using inducible Cas9, in association with targeting sgRNAs in pigs, which is more convenient compared with the conventional CRISPR/Cas9 system. Premature microRNAs (miRNAs) exist as a classical stem-loop structure. Therefore, DSBs could be generated using the CRISPR/Cas9 system. DSBs in the loop region may affect miRNA maturation during processing by Drosha and Dicer, resulting in knockdown of the miRNA in mammalian cells (33). Therefore, doxycycline-inducible FLAG-Cas9 PFFs were able be used for porcine genome editing and miRNA expression and regulation. The CRISPR-associated protein Cas9 not only identifies genomic DNA and generates sequence-specific dsDNA cleavage, but it also binds with high affinity to single-stranded RNA (ssRNA) targets matching the Cas9-associated guide RNA sequence when the PAM is presented in trans as a separate DNA oligonucleotide, and stimulates site-specific endonucleolytic cleavage of ssRNA targets, including endogenous mRNA with PAM-presenting oligonucleotides (PAMmers) (34). It is hypothesized that the 
A Sequences of inserted pCW-cas9

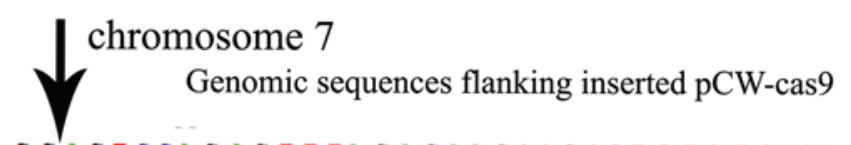

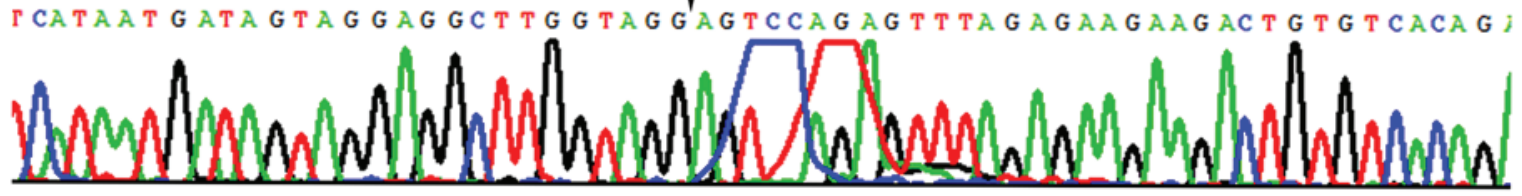

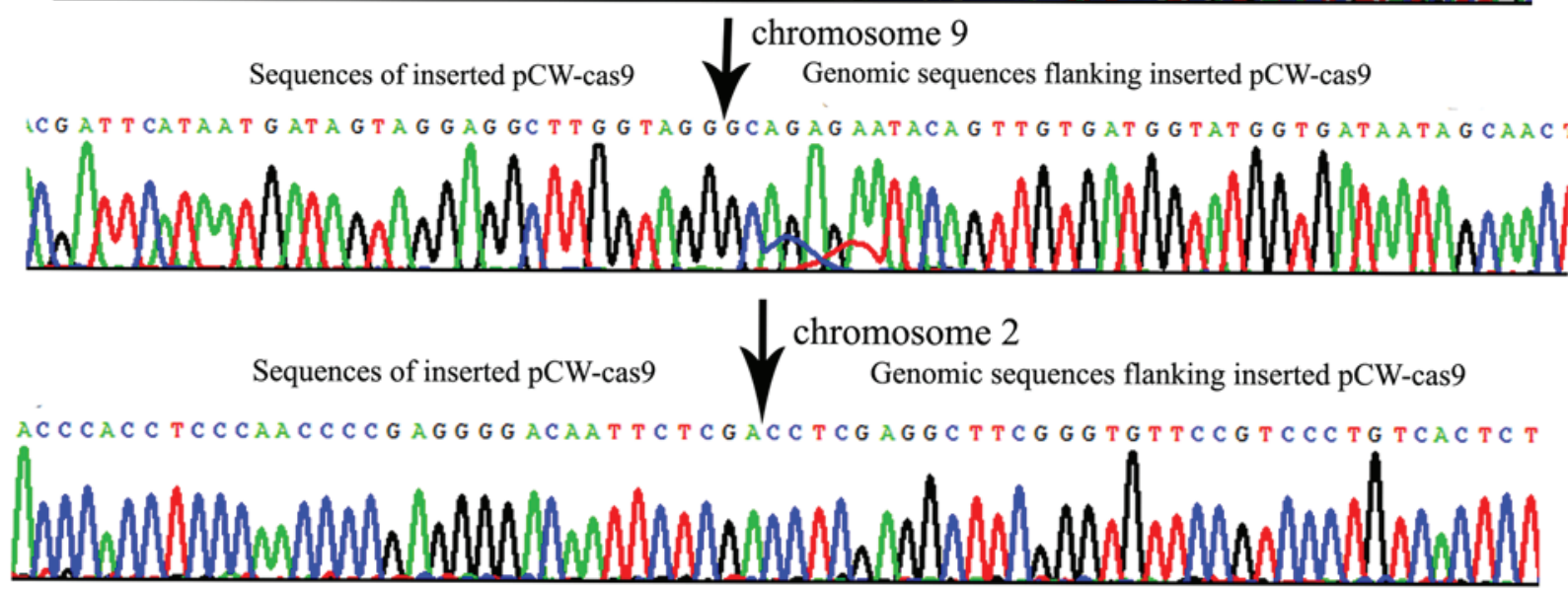

B

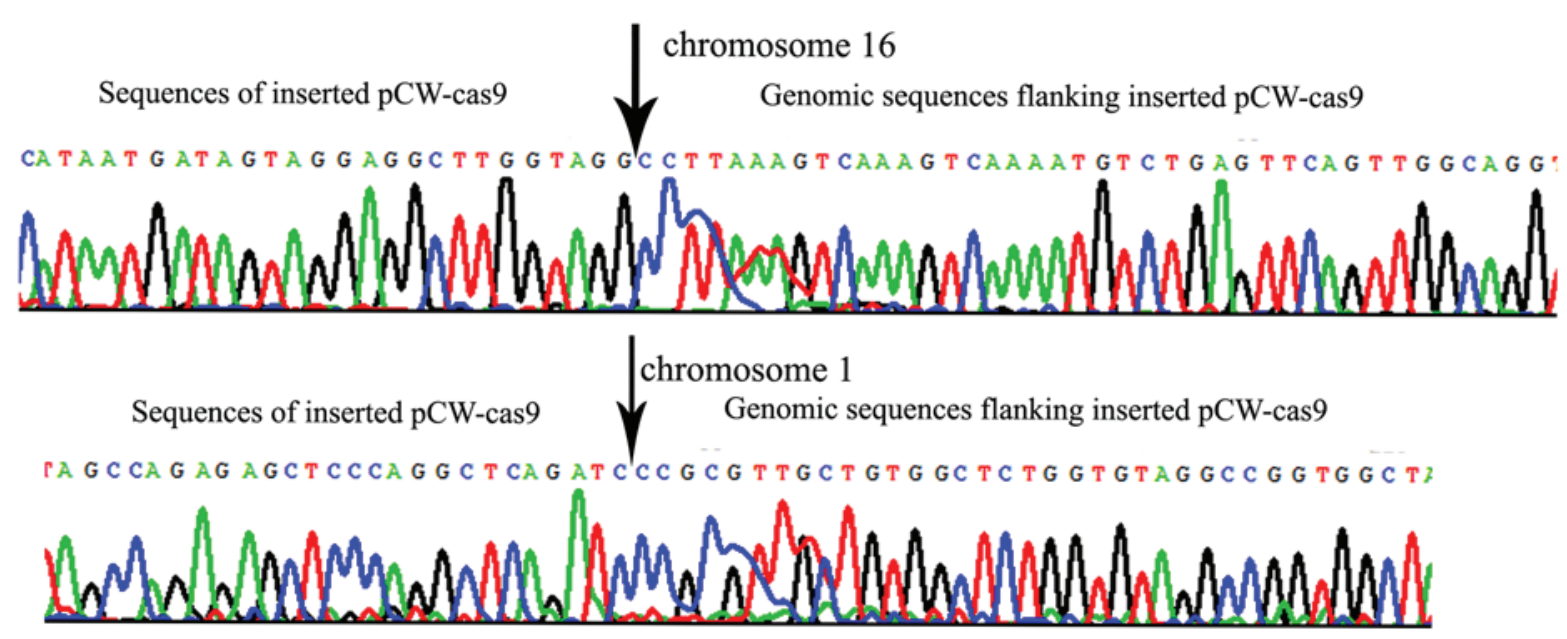

C

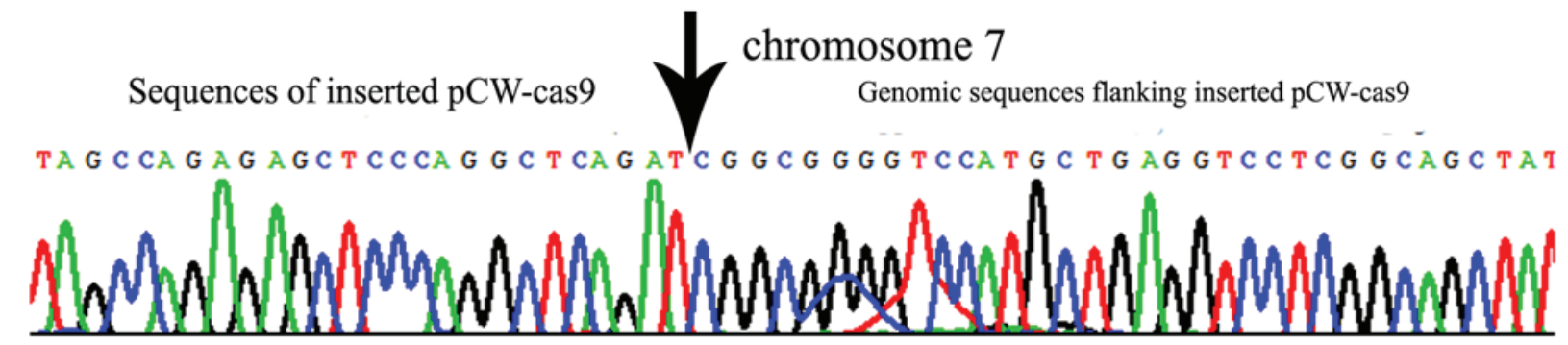

Figure 5. Identification of $\mathrm{pCW}$-cas9 integration sites in the transgenic PFF genome. Identified integration sites of pCW-Cas9 in the transgenic (A) PFF1, (B) PFF2 and (C) PFF3 genomes are shown. All the identified insertion site sequences contain the lentiviral vector LRT sequences, which is indicative of the insertion border between the lentiviral vector and flanking genomic DNA. PFF, porcine fetal fibroblast.

inducible Cas9 PFFs may be used to regulate porcine mRNA expression.

Loss-of-function screening is a powerful and hypothesis-free approach to identify genes and pathways that underlie biological processes. The CRISPR/Cas9 system was considered to be an ideal tool for genetic screening, since RNAi may only achieve a partial depletion of gene activity, and knockout-based screens are difficult to manage in diploid mammalian cells, for example, as has been applied to human and mouse cells $(17,18)$. PFFs are highly undifferentiated cells 
compared with other cells retrieved from adult tissue, and have been demonstrated to be the most effective donor cells for SCNT $(35,36)$. Therefore, the present study has revealed that a loss-of-function pig model may be generated on the basis of doxycycline-inducible flag-cas9 PFFs, and this function may be verified in vitro.

In conclusion, three porcine fibroblast cell lines that conditionally express Cas9 were established. These FLAG-Cas9 PFFs may be widely applicable in porcine genomic editing and the regulation of gene expression. In our future work, these cells are to be used with the intention of editing reproduction-associated genes in pigs. The porcine fibroblast cell lines expressing Cas 9 endonuclease may be productively used in SCNT procedures to generate a porcine model of genomic modification, along with targeting sgRNAs.

\section{Acknowledgements}

This study was supported by the National Basic Research and Development Program of China (973 Program, nos. 2011CB944202 and 2010CB945001) and the National Natural Science Foundation of China (no. 31402072).

\section{References}

1. Luo Y, Lin L, Bolund L, Jensen TG and Sørensen CB: Genetically modified pigs for biomedical research. J Inherit Metab Dis 35: 695-713, 2012.

2. Tector AJ and Ford ML: Designing donors: Nuclease-based genome editing in pigs. Am J Transplant 15: 3, 2015.

3. Tan W, Carlson DF, Lancto CA, Garbe JR, Webster DA, Hackett PB and Fahrenkrug SC: Efficient nonmeiotic allele introgression in livestock using custom endonucleases. Proc Natl Acad Sci USA 110: 16526-16531, 2013.

4. Urnov FD, Rebar EJ, Holmes MC, Zhang HS and Gregory PD: Genome editing with engineered zinc finger nucleases. Nat Rev Genet 11: 636-646, 2010.

5. Petersen B, Ahrens H, Herrmann D, Petkov SG, Frenzel A, Hauschild J, Lucas-Hahn A, Hassel P, Ziegler M, Baars W et al: Generation of HHO-1/HA20/GGTA1-ko pigs by using sleeping beauty transposon and zinc finger nucleases. Transgenic Res 23 : $861,2014$.

6. Tang L, Gonzalez R and Dobrinski I: Germline modification of domestic animals. Anim Reprod 12: 93-104, 2015.

7. Miller JC, Tan S, Qiao G, Barlow KA, Wang J, Xia DF, Meng X, Paschon DE, Leung E, Hinkley SJ, et al: A TALE nuclease architecture for efficient genome editing. Nat Biotechnol 29: 143-148, 2011.

8. Yao J, Huang J, Hai T, Wang X, Qin G, Zhang H, Wu R, Cao C, Xi JJ, Yuan Z and Zhao J: Efficient bi-allelic gene knockout and site-specific knock-in mediated by TALENs in pigs. Sci Rep 4: 6926, 2014.

9. Pan D, Yuan J, Li X, Feng C, Long C, Cui H, Wang F and $\mathrm{Xu}$ J: Efficient generation of GGTA1-null pigs via TALENs. Transgenic Res 22: 246, 2013.

10. Wiedenheft B, Sternberg SH and Doudna JA: RNA-guided genetic silencing systems in bacteria and archaea. Nature 482 : 331-338, 2012

11. Jinek M, Chylinski K, Fonfara I, Hauer M, Doudna JA and Charpentier E: A programmable dual-RNA-guided DNA endonuclease in adaptive bacterial immunity. Science 337: 816-821, 2012.

12. Sternberg SH, Redding S, Jinek M, Greene EC and Doudna JA DNA interrogation by the CRISPR RNA-guided endonuclease Cas9. Nature 507: 62-67, 2014.

13. Hai T, Teng F, Guo R, Li W and Zhou Q: One-step generation of knockout pigs by zygote injection of CRISPR/Cas system. Cell Res 24: 372-375, 2014.

14. Whitworth KM, Lee K, Benne JA, Beaton BP, Spate LD, Murphy SL, Samuel MS, Mao J, O'Gorman C, Walters EM, et al: Use of the CRISPR/Cas9 system to produce genetically engineered pigs from in vitro-derived oocytes and embryos. Biol Reprod 91: 78, 2014.
15. Zhou X, Xin J, Fan N, Zou Q, Huang J, Ouyang Z, Zhao Y, Zhao B, Liu Z, Lai S, et al: Generation of CRISPR/Cas9-mediated gene-targeted pigs via somatic cell nuclear transfer. Cell Mol Life Sci 72: 1175-1184, 2015.

16. Wang T, Wei JJ, Sabatini DM and Lander ES: Genetic screens in human cells using the CRISPR-Cas9 system. Science 343: 80-84, 2014.

17. Shalem O, Sanjana NE, Hartenian E, Shi X, Scott DA, Mikkelsen TS, Heckl D, Ebert BL, Root DE, Doench JG and Zhang F: Genome-Scale CRISPR-Cas9 knockout screening in human cells. Science 343: 84-87, 2014.

18. Koike-Yusa H, Li Y, Tan EP, Velasco-Herrera Mdel C and Yusa K: Genome-wide recessive genetic screening in mammalian cells with a lentiviral CRISPR-guide RNA library. Nat Biotechnol 32: 267-273, 2014.

19. Doench JG, Hartenian E, Graham DB, Tothova Z, Hegde M, Smith I, Sullender M, Ebert BL, Xavier RJ and Root DE: Rational design of highly active sgRNAs for CRISPR-Cas9-mediated gene inactivation. Nat Biotechnol 32: 1262-1267, 2014.

20. Wu Z, Xu Z, Zou X, Zeng F, Shi J, Liu D, Urschitz J, Moisyadi S and Li Z: Pig transgenesis by piggyBac transposition in combination with somatic cell nuclear transfer. Transgenic Res 22: 1107-1118, 2013.

21. Deng W, Yang D, Zhao B, Ouyang Z, Song J, Fan N, Liu Z, Zhao Y, Wu Q, Nashun B, et al: Use of the $2 \mathrm{~A}$ peptide for generation of multi-transgenic pigs through a single round of nuclear transfer. PLoS One 6: e19986, 2011.

22. Kues WA, Petersen B, Mysegades W, Carnwath JW and Niemann $\mathrm{H}$ : Isolation of murine and porcine fetal stem cells from somatic tissue. Biol Reprod 72: 1020-1028, 2005.

23. Zor T and Selinger Z: Linearization of the Bradford protein assay increases its sensitivity: theoretical and experimental studies. Anal Biochem 236: 302-308, 1996.

24. Cheng T, Xue X and Fu J: Effect of OLIG1 on the development of oligodendrocytes and myelination in a neonatal rat PVL model induced by hypoxia-ischemia. Mol Med Rep 11: 2379-2386, 2015.

25. Gasiunas G, Barrangou R, Horvath P and Siksnys V: Cas9-crRNA ribonucleoprotein complex mediates specific DNA cleavage for adaptive immunity in bacteria. Proc Natl Acad Sci USA 109: E2579-E2586, 2012.

26. Yang M, Zhang L, Stevens J and Gibson G: CRISPR/Cas9 mediated generation of stable chondrocyte cell lines with targeted gene knockouts; Analysis of an aggrecan knockout cell line. Bone 69: 118-125, 2014.

27. Kim B, Jin D, Kim H, Kim M and Park C: Analysis of transgene intergration efficiency into porcine fetal fibroblast using different transfection methods. Reprod Dev Biol 33: 113-117, 2009.

28. Blanton JR Jr, Bidwell CA, Sanders DA, Sharkey CM, McFarland DC, Gerrard DE and Grant AL: Plasmid transfection and retroviral transduction of porcine muscle cells for cell-mediated gene transfer. J Anim Sci 78: 909-918, 2000.

29. Niu Y, Shen B, Cui Y, Chen Y, Wang J, Wang L, Kang Y, Zhao X, Si W, Li W, et al: Generation of gene-modified cynomolgus monkey via Cas9/RNA-mediated gene targeting in one-cell embryos. Cell 156: 836-843, 2014.

30. Shen B, Zhang J, Wu H, Wang J, Ma K, Li Z, Zhang X, Zhang $P$ and Huang $X$ : Generation of gene-modified mice via Cas9/RNA-mediated gene targeting. Cell Res 23: 720-723, 2013.

31. Shao Y, Guan Y, Wang L, Qiu Z, Liu M, Chen Y, Wu L, Li Y, Ma X, Liu M and Li D: CRISPR/Cas-mediated genome editing in the rat via direct injection of one-cell embryos. Nat Protoc 9: 2493-2512, 2014.

32. Li P, Estrada JL, Burlak C, Montgomery J, Butler JR, Santos RM, Wang ZY, Paris LL, Blankenship RL, Downey SM, et al: Efficient generation of genetically distinct pigs in a single pregnancy using multiplexed single-guide RNA and carbohydrate selection. Xenotransplantation 22: 20-31, 2015.

33. Zhao Y, Dai Z, Liang Y, Yin M, Ma K, He M, Ouyang H and Teng CB: Sequence-specific inhibition of microRNA via CRISPR/CRISPRi system. Sci Rep 4: 3943, 2014.

34. O'Connell MR, Oakes BL, Sternberg SH, East-Seletsky A, Kaplan M and Doudna JA: Programmable RNA recognition and cleavage by CRISPR/Cas9. Nature 516: 263-266, 2014.

35. Lee GS, Hyun SH, Kim HS, Kim DY, Lee SH, Lim JM, Lee ES, Kang SK, Lee BC and Hwang WS: Improvement of a porcine somatic cell nuclear transfer technique by optimizing donor cell and recipient oocyte preparations. Theriogenology 59: 1949-1957, 2003.

36. Wei H, Qing Y, Pan W, Zhao H, Li H, Cheng W, Zhao L, Xu C, $\mathrm{Li} \mathrm{H}, \mathrm{Li}$ S, et al: Comparison of the efficiency of Banna miniature inbred pig somatic cell nuclear transfer among different donor cells. PLOS One 8: e57728, 2013. 